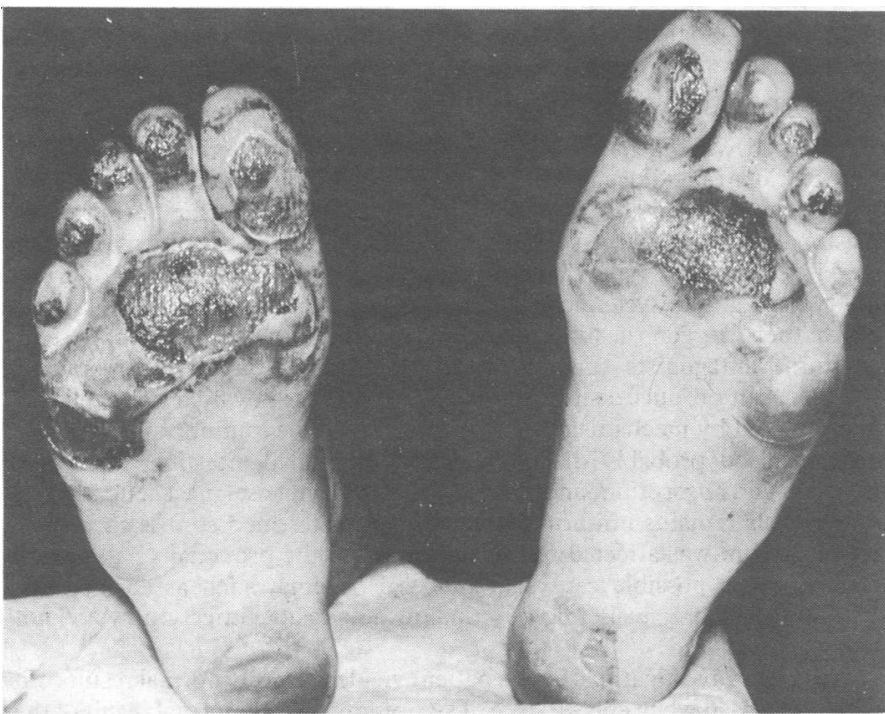

Partial thickness loss of skin on feet.

Although this problem has not been described before in runners, experimental and clinical studies have shown that nerve conduction is altered by cooling. Ungley $e t$ al looked at the symptoms experienced by naval staff during the second world war after immersion of their feet in cold sea water: all the subjects reported loss of sensation.' Sinclair $e t$ al showed that cooling of the legs results in, firstly, cold feet, then pain, then loss of sensation to touch. ${ }^{2}$ In a more recent study Basbaum cooled the sciatic nerve of a rat and found that a reduction in the temperature of the nerve to $5^{\circ} \mathrm{C}$ resulted in a total block in conduction, which recovered on warming. ${ }^{3}$

It is likely that because of the low ground temperature $\left(-9^{\circ} \mathrm{C}\right)$ the runners reported on here developed temporary neuropathy of the feet, lost all sensation, and were able to continue running despite the severity of their injuries. This indicates the dangers of running on cold surfaces without adequate footware.

1 Ungley CC, Channell GD, Richards RL. Immersion foot syndrome. Brf Sirg 1945;33:17-31. 2 Sinclair DC, Hinshaw JR. Sensory changes in nerve blocks induced by cooling. Brain 1951;74 318-35.

3 Basbaum CB. Induced hypothermia in peripheral nerve: electron microscopic and electrophysiological observations. I Neurocyrol 1973;2:171-87.

(Accepted 28 October 1986)

Leicester Royal Infirmary, Leicester LE1 5WW

M REICHL, FRCSED, accident and emergency registrar

\section{Occult chlamydial ophthalmia in men with non-gonococcal urethritis}

Chlamydial ophthalmia in adults usually results from autoinnoculation with genital secretion containing Chlamydia trachomatis (D-K). ${ }^{12}$ Little is known about the relation between occult chlamydial ophthalmia and overt genitourinary infections. The recent introduction of fluorescein labelled monoclonal antibody allows rapid and reliable identification of chlamydia in conjunctival smears. ${ }^{3}$ We used this method in a prospective study to evaluate the prevalence of chlamydia in the eye in men with urethritis associated with chlamydia.

\section{Patients, methods, and results}

Informed consent to ophthalmological assessment was given by 60 out of 67 men with non-gonococcal urethritis who presented consecutively to the department of genitourinary medicine. The patients' eyes were examined by slit lamp for conjunctival papillas and follicles, corneal staining, and opacities. Papillas and follicles in both eyes were graded on a four point scale. ${ }^{4}$

Topical amethocaine $1 \%$ was instilled in both eyes, and after five minutes conjunctival scrapings were taken with a scalpel blade, smeared on to a microscope slide, and fixed with methanol. Smears were stained with Orion Chlamyset fluorescein labelled monoclonal antibody and the fluorescent chlamydial inclusion bodies counted $C$ trachomatis was isolated from urethral swabs by innoculation on to McCoy cell monolayers treated with idoxuridine. The data were analysed by $\chi^{2}$ (with Yates's correction), Student's $t$ test, and analysis of variance (one way).

Eight of the 60 men had asymptomatic chlamydial ophthalmia; none had punctate keratitis, pannus, or tarsal scarring. Conjunctival smears showed five to 10 inclusion bodies in five men and 10-50 in the remaining three. Six of the eight men had evidence of genital infection associated with chlamydia as indicated by isolation of chlamydia either in the man or in his consort. There were no significant demographic or clinical differences between those who had chlamydia in the eye and those who did not (table). The eye disease was apparently cured by conventional treatment with oral triple tetracycline (tetracycline, chlortetracycline, and demeclocycline; Deteclo) for two weeks.

Epidemiological and clinical features of men with non-gonococcal urethritis

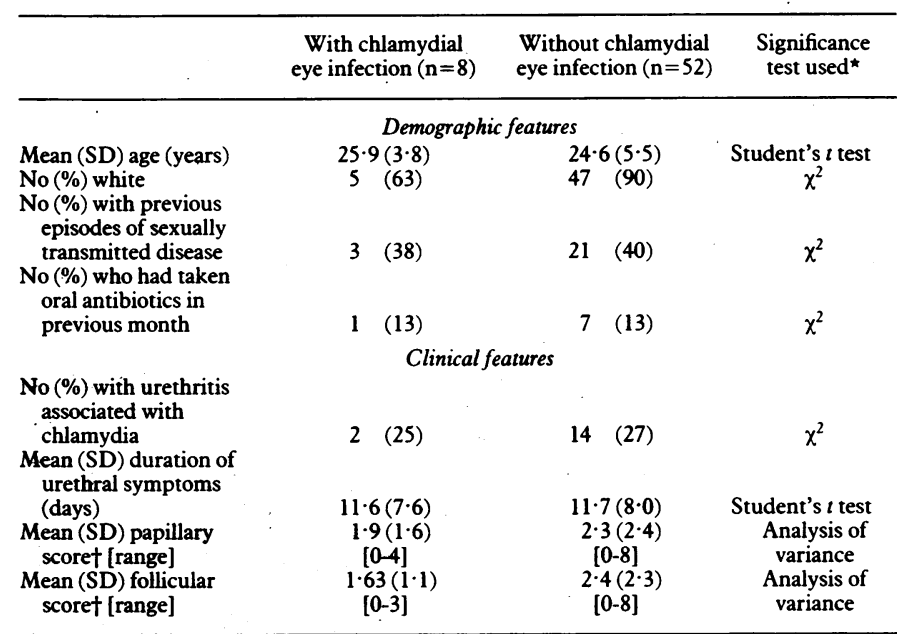

*All differences in variables between groups not significant.

† Sum of grades of papillas or follicles on upper and lower eyelids in both eyes $(0=$ none, $1=$ mild, $2=$ moderate, $3=$ severe; maximum score $=12$ ).

\section{Comment}

Asymptomatic chlamydial eye infection in men with non-gonococcal urethritis has not been described previously; indeed, in Sweden a study of cultures failed to identify any cases of chlamydial eye infection in $\mathbf{8 5 5}$ unselected patients attending a clinic for sexually transmitted diseases, even though $18 \cdot 6 \%$ had genital chlamydia. ${ }^{5}$ In our study those patients who had chlamydia in the eye had few inclusion bodies. We postulate that the technique of conjunctival scraping combined with use of a specific monoclonal antibody to detect $C$ trachomatis is more sensitive than smears and conventional tissue culture. The minor conjunctival changes seen, predominantly papillas and follicles, were common and did not distinguish clinically between those who did and did not have chlamydial eye infection. This parallels the well recognised occult nature of chlamydial infection of the genital tract.

This study shows that chlamydial eye disease may be asymptomatic and is not uncommon in men with non-gonococcal urethritis. Further studies are necessary to document the clinical course of this condition and its response to concurrent treatment of urethritis associated with chlamydia.

1 Viswalingam ND, Wishart MS, Woodland RM. Adult chlamydial ophthalmia. Br Med Bull 1983;39:123-7.

2 Dunlop EMC, Jones BR, AI Hussaini MK. Genital infection in association with TRIC virus infection of the eye. Br $\mathcal{F}$ Vener Dis 1964;40:33-42.

3 Hawkins DA, Wilson RS, Thomas BJ, Evans RT. Rapid, reliable diagnosis of chlamydial ophthalmia by means of monoclonal antibodies. Brf Ophthalmol 1985;69:640-4.

4 Dawson CR, Jones BR, Darougar S. Blinding and non blinding trachoma, the assessment of intensity of upper tarsal inflammatory disease and blinding lesions. Bull WHO 1975;52:279-82. 5 Ronnerstam R, Persson K, Hansson $H$, Renmarker $K$. Prevalence of chlamydial eye infections in patients attending an eye clinic, a VD clinic and in healthy persons. $\mathrm{Br} \mathcal{J}$ Ophthalmol 1985;69:385-8.

(Accepted 21 October 1986)

Department of Genitourinary Medicine and Ophthalmology, Royal Hallamshire Hospital, Sheffield S10 2JF

E F MONTEIRO, MRCP, senior registrar in genitourinary medicine

J A BRADBURY, FRCS, registrar in ophthalmology

M O'DONNELL, FRCS, registrar in ophthalmology

I G RENNIE, FRCS, senior lecturer in ophthalmology

G R KINGHORN, MD, MRCP, consultant in genitourinary medicine

Correspondence to: Dr Monteiro. 